\title{
Detection of interstellar hydrogen peroxide ${ }^{\star}$
}

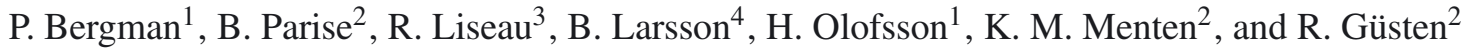 \\ 1 Onsala Space Observatory, Chalmers University of Technology, 43992 Onsala, Sweden \\ e-mail: per . bergman@chalmers.se \\ 2 Max Planck Institut für Radioastronomie, Auf dem Hügel 69, 53121 Bonn, Germany \\ 3 Department of Earth and Space Sciences, Chalmers University of Technology, 43992 Onsala, Sweden \\ ${ }^{4}$ Department of Astronomy, Stockholm University, AlbaNova, 10691 Stockholm, Sweden
}

Received 2 May 2011 / Accepted 25 May 2011

ABSTRACT

\begin{abstract}
Context. The molecular species hydrogen peroxide, $\mathrm{HOOH}$, is likely to be a key ingredient in the oxygen and water chemistry in the interstellar medium.

Aims. Our aim with this investigation is to determine how abundant $\mathrm{HOOH}$ is in the cloud core $\rho$ Oph A.

Methods. By observing several transitions of $\mathrm{HOOH}$ in the (sub)millimeter regime we seek to identify the molecule and also to determine the excitation conditions through a multilevel excitation analysis.

Results. We have detected three spectral lines toward the SM1 position of $\rho$ Oph A at velocity-corrected frequencies that coincide very closely with those measured from laboratory spectroscopy of $\mathrm{HOOH}$. A fourth line was detected at the $4 \sigma$ level. We also found through mapping observations that the $\mathrm{HOOH}$ emission extends (about $0.05 \mathrm{pc}$ ) over the densest part of the $\rho$ Oph A cloud core. We derive an abundance of $\mathrm{HOOH}$ relative to that of $\mathrm{H}_{2}$ in the SM1 core of about $1 \times 10^{-10}$.

Conclusions. To our knowledge, this is the first reported detection of $\mathrm{HOOH}$ in the interstellar medium.
\end{abstract}

Key words. astrochemistry - ISM: abundances - ISM: individual objects: $\rho$ Oph A - ISM: molecules

\section{Introduction}

Hydrogen peroxide, $\mathrm{HOOH}$, is believed to play an important role in the Earth's atmospheric ozone and water chemistry. It is a key constituent in the gas- and liquid-phase radical chemistry and has an oxidizing potential in the liquid phase. Gas-phase $\mathrm{HOOH}$ has been seen in the Martian atmosphere by ground-based observations (Clancy et al. 2004; Encrenaz et al. 2004). However, recent Mars observations with the Herschel Observatory (Hartogh et al. 2010) failed to detect $\mathrm{HOOH}$ at levels below those previously seen. The non-detection was attributed to seasonal variations.

Interestingly, $\mathrm{HOOH}$ is among the simplest molecules that show internal rotation. The internal rotation, or torsion, manifests itself as a rotation of the two $\mathrm{O}-\mathrm{H}$ bonds about the $\mathrm{O}-\mathrm{O}$ bond. This hindered internal rotation can be described with a torsion potential in which the two minima (the most stable configurations) do not coincide with the cis or trans alignment of the two $\mathrm{O}-\mathrm{H}$ bonds ${ }^{1}$. The twofold barrier gives rise to a quartet of sublevels for each torsional state. These sublevels are denoted $\tau=1,2,3,4$ (Hunt et al. 1965). Moreover, $\mathrm{HOOH}$ is a light, slightly asymmetric prolate rotor (belonging to the $C_{2 h}^{\dagger}$ point group, see Hougen 1984, for a discussion), with only $c$-type

\footnotetext{
* Based on observations with the Atacama Pathfinder EXperiment (APEX) telescope. APEX is a collaboration between the Max-PlanckInstitut für Radioastronomie, the European Southern Observatory, and the Onsala Space Observatory.

1 When the two O-H bonds point in the same direction, this is referred to as the cis position, while the 180 degree opposite case is called the trans position. For $\mathrm{HOOH}$, the trans potential barrier height is $557 \mathrm{~K}$, while the cis barrier height is almost $4000 \mathrm{~K}$ (Pelz et al. 1993).
}

transitions and with a dipole moment of $1.6 \mathrm{D}$ (Cohen \& Pickett 1981; Perrin et al. 1996). Because of this, no pure rotational transitions occur, and only transitions corresponding to a combined ro-torsional motion change can take place. The $\mathrm{mm}$ and submm spectrum of $\mathrm{HOOH}$ has been studied rather extensively in the laboratory (Helminger et al. 1981; Petkie et al. 1995) and is available in the JPL database (Pickett et al. 1998).

To our knowledge, $\mathrm{HOOH}$ has not so far been detected in the interstellar medium. Also, very few abundance limits have been reported. Blake et al. (1987) derived an upper limit for $\mathrm{HOOH}$ of $4.5 \times 10^{-10}$ with respect to $\mathrm{H}_{2}$ from their Orion spectral scan data. Boudin et al. (1998) reported an upper limit of $5.2 \%$ of solid $\mathrm{HOOH}$ relative to $\mathrm{H}_{2} \mathrm{O}$ ice toward NGC 7538 IRS9.

As in the Earth's atmosphere, $\mathrm{HOOH}$ is expected to be closely connected to the water and molecular oxygen chemistry also for those physical conditions prevailing in molecular clouds. In current pure gas-phase models, $\mathrm{HOOH}$ is formed by reaction of $\mathrm{H}_{2}$ with $\mathrm{HO}_{2}$ or via the reaction involving two $\mathrm{OH}$ radicals. However, these reactions proceed very slowly ${ }^{2}$. Alternatively, Tielens \& Hagen (1982) suggested that $\mathrm{HOOH}$ could be formed on grain surfaces by the successive additions of $\mathrm{H}$ atoms to molecular oxygen. If this is the case, $\mathrm{HOOH}$ could be closely related to the amount of $\mathrm{O}_{2}$ on grains.

The $119 \mathrm{GHz}$ line of $\mathrm{O}_{2}$ was detected toward the cloud $\rho$ Oph A with an abundance of $5 \times 10^{-8}$ relative to $\mathrm{H}_{2}$ by Larsson et al. (2007) using the Odin satellite. The $\rho$ Oph A molecular cloud, at a distance of about $120 \mathrm{pc}$, has been the subject of several studies. Continuum observations (André et al. 1993; Motte et al. 1998) and $\mathrm{C}^{18} \mathrm{O}$ observations (Liseau et al. 2010) revealed

\footnotetext{
${ }^{2}$ http://www.physics.ohio-state.edu/ eric
} 
Table 1. Observed $\mathrm{HOOH}$ lines.

\begin{tabular}{lcccc}
\hline \hline $\begin{array}{l}\text { Frequency } \\
(\mathrm{MHz})\end{array}$ & \multicolumn{2}{c}{ Transition } & $E_{\mathrm{u}}$ & $A_{\mathrm{ul}}$ \\
& $J_{K_{\mathrm{a}}^{\prime}, K_{\mathrm{c}}^{\prime}}^{\prime}-J_{K_{\mathrm{a}}^{\prime \prime}, K_{\mathrm{c}}^{\prime \prime}}^{\prime \prime}$ & $\tau^{\prime}-\tau^{\prime \prime}$ & $(\mathrm{K})$ & $\left(\mathrm{s}^{-1}\right)$ \\
\hline 219166.86 & $3_{0,3}-2_{1,1}$ & $4-2$ & 31.2 & $8.58 \times 10^{-5}$ \\
251914.68 & $6_{1,5}-5_{0,5}$ & $2-4$ & 65.5 & $2.46 \times 10^{-4}$ \\
268961.17 & $4_{0,4}-3_{1,2}$ & $4-2$ & 41.1 & $1.84 \times 10^{-4}$ \\
318222.52 & $5_{0,5}-4_{1,3}$ & $4-2$ & 53.4 & $3.31 \times 10^{-4}$ \\
318712.10 & $5_{1,4}-6_{0,6}$ & $3-1$ & 67.0 & $4.12 \times 10^{-4}$ \\
670595.82 & $1_{1,0}-0_{0,0}$ & $3-1$ & 32.2 & $5.79 \times 10^{-3}$ \\
\hline
\end{tabular}

several cores. Very recently, Bergman and coworkers found a very high degree of deuteration toward the SM1 core in $\rho$ Oph A from observations of deuterated $\mathrm{H}_{2} \mathrm{CO}$ (Bergman et al. 2011). These observations suggest that grain surface reactions were at work to produce the very high deuterium levels observed in the gas-phase material.

In this Letter we continue our study of the $\rho$ Oph A cloud by reporting on observations of several $\mathrm{HOOH}$ transitions. We have detected four $\mathrm{HOOH}$ lines and two of these lines were mapped over the central part of this cloud. In Sect. 2 we describe our observations and present our results. There, we also describe in more detail the energy level structure and symmetry of $\mathrm{HOOH}$, which is important for discussing the detected lines. Then, in Sect. 3, we discuss the implications of our results.

\section{Observations and results}

We have used the APEX $12 \mathrm{~m}$ telescope located at about $5100 \mathrm{~m}$ altitude in the Chilean Andes (Güsten et al. 2006) to observe $\mathrm{HOOH}$. For the lower frequency lines we used the Swedish heterodyne facility instruments APEX-1 and APEX-2 (Vassilev et al. 2008). The 7-pixel longer wavelength $(450 \mu \mathrm{m})$ module of the MPIfR-built CHAMP ${ }^{+}$receiver array (Kasemann et al. 2006) was used for the observations of a high-frequency line. The 7 pixels, spaced by $18^{\prime \prime}$, are arranged in a hexagon around a central pixel. The observations took place on several occasions during 2010; April 2-11, July 7, August 4-8, and September 10-13.

The targeted $\mathrm{HOOH}$ lines are listed in Table 1. The frequency, transition quantum number designation, energy of upper level, and Einstein $A$-coefficient are listed. All values have been compiled from the JPL catalogue (Pickett et al. 1998). The frequency uncertainty for the listed lines is $0.1 \mathrm{MHz}$ or better and this corresponds to $0.14 \mathrm{~km} \mathrm{~s}^{-1}$ at $219 \mathrm{GHz}$. We also present in Fig. 1 the energy diagrams for all levels below $100 \mathrm{~K}$. Owing to the symmetry of $\mathrm{HOOH}$, four different radiatively decoupled ladders occur: $A_{1 \leftrightarrow 3}, A_{2 \leftrightarrow 4}, B_{1 \leftrightarrow 3}$, and $B_{2 \leftrightarrow 4}$. The subscript indicates the pair of torsional quantum numbers $\tau$ involved. The $c$-type electric dipole transitions must also obey $\tau=1 \leftrightarrow 3$ or $\tau=2 \leftrightarrow 4$ (Hunt et al. 1965) and are drawn as downward arrows connecting the upper and lower levels in Fig. 1. The $A$-species have a nuclear spin weight of 1 and the $B$-species have a spin weight of 3 (Hougen 1984). This is, of course, due to the nuclear spin directions of the two $\mathrm{H}$ atoms (in the same way as the ortho and para symmetries occur for $\mathrm{H}_{2} \mathrm{O}$ or $\mathrm{H}_{2} \mathrm{CO}$ ).

The ground-state symmetry species is that of $A_{1 \leftrightarrow 3}$ with the $B_{1 \leftrightarrow 3}$ state only about $2.5 \mathrm{~K}$ higher energy. This means that the energy difference between the species with different nuclear spin weights is much smaller than the corresponding difference for $\mathrm{H}_{2} \mathrm{O}$ or $\mathrm{H}_{2} \mathrm{CO}$. The torsional $\tau=2,4$ states are about $16 \mathrm{~K}$ above the $\tau=1,3$ states. This difference stems from the tunneling through the trans barrier and is comparable to the gas kinetic
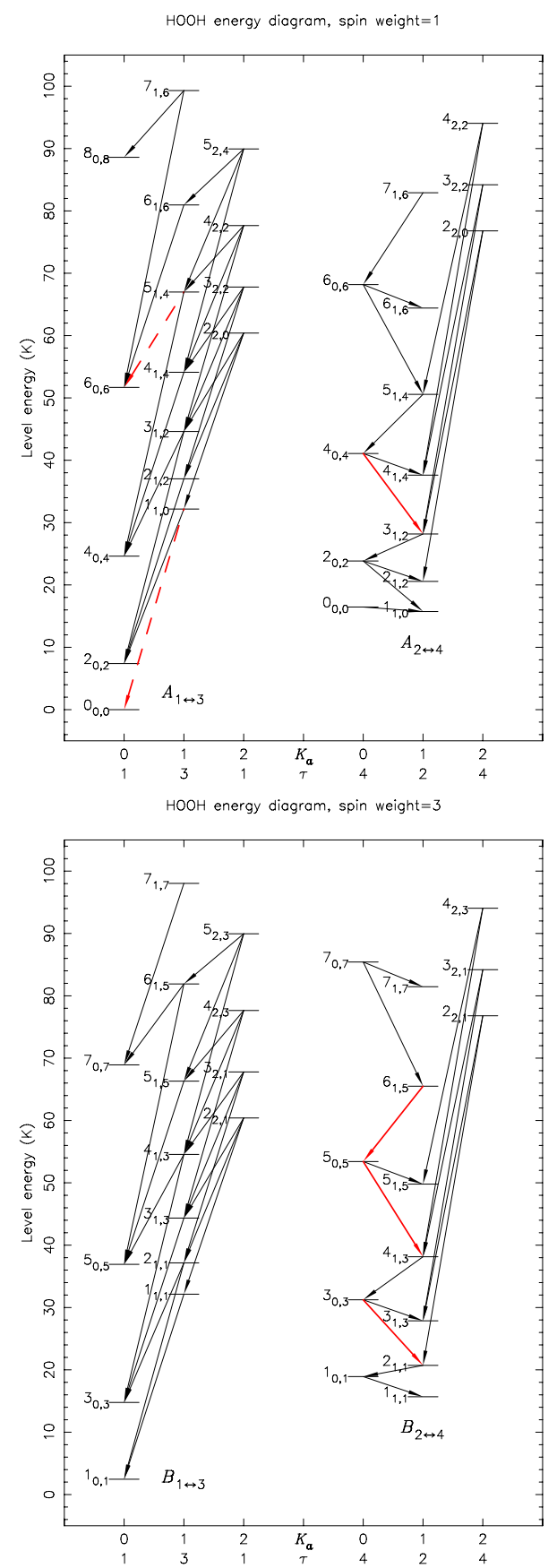

Fig. 1. HOOH energy level diagrams. The energy is given in $K$ on the vertical axis, and at the bottom the quantum numbers $K_{a}$ and $\tau$ are shown. To the left of each level the rotational quantum numbers $J_{K_{a}, K_{c}}$ are listed. The upper diagram shows the levels with a nuclear spin weight of $1\left(A_{1 \leftrightarrow 3}\right.$ and $\left.A_{2 \leftrightarrow 4}\right)$, while those levels with a spin weight of $3\left(B_{1 \leftrightarrow 3}\right.$ and $\left.B_{2 \leftrightarrow 4}\right)$ are shown in the lower diagram. The allowed $c$-type transitions within each of the four ladders are indicated as arrows. The red arrows represent detected lines, while the red-dashed arrows indicate non-detections.

temperatures of 20-30 K found in $\rho$ Oph A (Loren et al. 1990; Liseau et al. 2003; Bergman et al. 2011).

In Fig. 2 we show the $\mathrm{HOOH}$ spectra toward the core of $\rho$ Oph A. The upper five spectra are toward the SM1 core, $\alpha(\mathrm{J} 2000)=16^{\mathrm{h}} 26^{\mathrm{m}} 27.2^{\mathrm{s}}$ and $\delta(\mathrm{J} 2000)=-24^{\circ} 24^{\prime} 04^{\prime \prime}$. The $670 \mathrm{GHz} \mathrm{CHAMP}^{+}$spectrum is an average of all pixels and is centered 30" north of the SM1 position (usually denoted SM1N). In Fig. 3 spectra of the $219166 \mathrm{MHz}$ line for the 
P. Bergman et al.: Detection of interstellar hydrogen peroxide

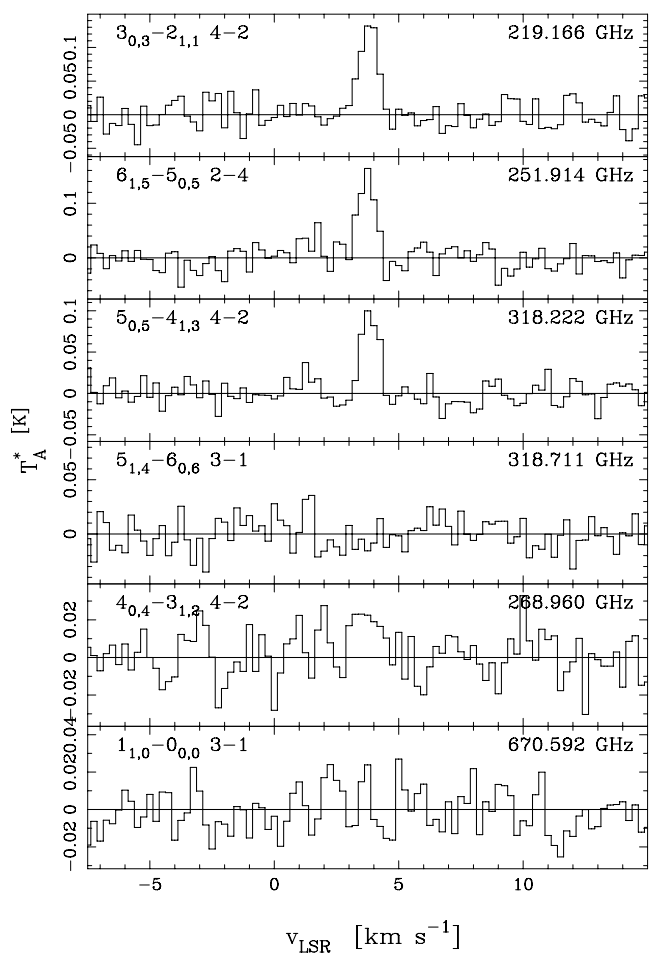

Fig. 2. HOOH spectra toward $\rho$ Oph A. The transition is indicated in each spectrum. The $T_{\mathrm{A}}^{*}$ intensity scale is in $K$ and the velocity $\left(v_{\mathrm{LSR}}\right)$ scale is in $\mathrm{km} \mathrm{s}^{-1}$. The velocity resolution is $0.25 \mathrm{~km} \mathrm{~s}^{-1}$.

Table 2. Observed $\mathrm{HOOH}$ line velocities, widths, and intensities.

\begin{tabular}{|c|c|c|c|c|}
\hline $\begin{array}{l}\text { Freq. } \\
(\mathrm{MHz})\end{array}$ & $\begin{array}{c}\text { Beam size } \\
(\operatorname{arcsec})\end{array}$ & $\begin{array}{c}v_{\mathrm{LSR}} \\
\left(\mathrm{km} \mathrm{s}^{-1}\right)\end{array}$ & $\begin{array}{l}F W H M \\
\left(\mathrm{~km} \mathrm{~s}^{-1}\right)\end{array}$ & $\underset{\left(\mathrm{K} \mathrm{km} \mathrm{s}^{-1}\right)}{T_{\mathrm{mb}} \mathrm{d} v^{a}}$ \\
\hline 219167 & 28 & 3.8 & 0.84 & $0.167(0.018)$ \\
\hline 251915 & 25 & 3.7 & 0.75 & $0.165(0.018)$ \\
\hline 268961 & 23 & 3.7 & (1.2) & $0.040(0.011)$ \\
\hline 318223 & 20 & 3.8 & 0.78 & $0.106(0.013)$ \\
\hline 318712 & 20 & & & $<0.044$ \\
\hline 670596 & 9 & & & $\begin{array}{l}<0.12^{b} \\
<0.051^{c}\end{array}$ \\
\hline
\end{tabular}

Notes. ${ }^{(a)}$ Integr. from 2.5 to $4.5 \mathrm{~km} \mathrm{~s}^{-1}$, errors are $1 \sigma$, upper limits are $3 \sigma ;{ }^{(b)}$ average value from three pixels closest to the SM1 position; (c) average value from all pixels, central pixel on SM1N.

observed map positions are shown. In the southern part the line is centered at $3.7 \mathrm{~km} \mathrm{~s}^{-1}$, while further north, at offset $\left(0^{\prime \prime},+60^{\prime \prime}\right)$, the line is at $3.2 \mathrm{~km} \mathrm{~s}^{-1}$. This NS velocity gradient is almost identical to the one seen for $\mathrm{H}_{2} \mathrm{CO}$ and its deuterated variants (Bergman et al. 2011). The narrow peaks to the NW are adjacent to where the sulphur species peak as noted by the same authors. In addition to the map in Fig. 3, we also mapped the $251 \mathrm{GHz}$ line, albeit with a poorer $\mathrm{S} / \mathrm{N}$ ratio and will not discuss it further here.

The beam sizes, velocity-integrated main-beam brightness temperatures $\left(\int T_{\mathrm{mb}} \mathrm{d} v\right)$, fitted LSR velocities $\left(v_{\mathrm{LSR}}\right)$, and line widths $(F W H M)$ are tabulated in Table 2. The $T_{\mathrm{mb}}$-scale was established assuming main-beam efficiencies of $0.75,0.73$, and 0.4 , for APEX-1, 2, and $\mathrm{CHAMP}^{+}$, respectively. The upper limits are $3 \sigma$ and for the $\mathrm{CHAMP}^{+}$line two intensities are listed in Table 2; one for the three pixels closest to the SM1 position and the other by averaging data from all pixels. The tabulated errors and upper limits depend only on the channel noise.

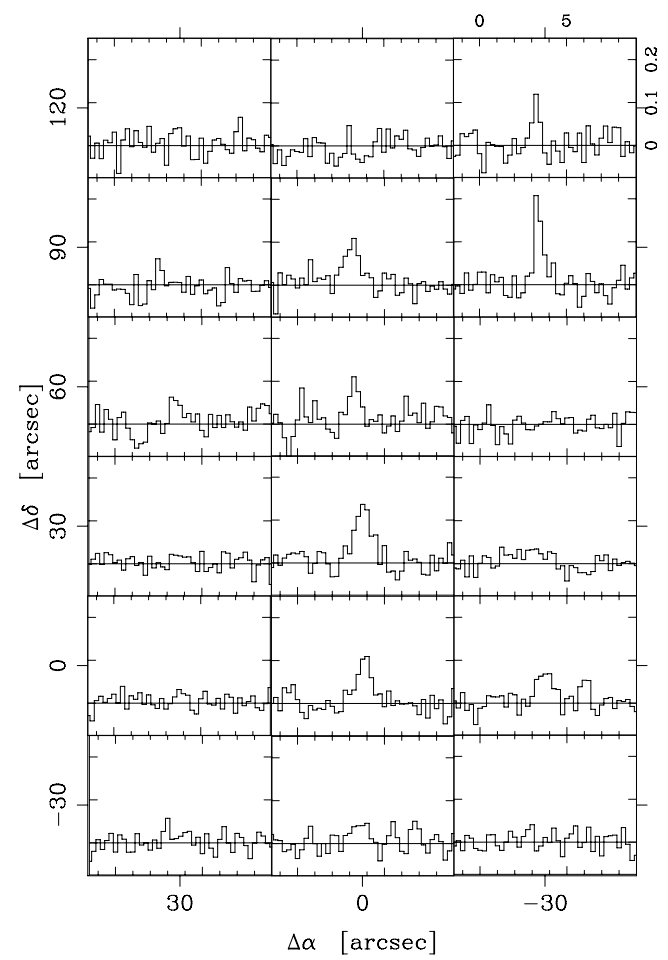

Fig. 3. Map spectra of the $219166 \mathrm{MHz} \mathrm{HOOH}$ line toward $\rho$ Oph A. The map offset $\left(0^{\prime \prime}, 0^{\prime \prime}\right)$ corresponds to the SM1 position. The $T_{\mathrm{A}}^{*}$ intensity scale, in $\mathrm{K}$, and the velocity $\left(v_{\mathrm{LSR}}\right) \mathrm{scale}$, in $\mathrm{km} \mathrm{s}^{-1}$, are indicated in the upper right spectrum. The velocity resolution is $0.25 \mathrm{~km} \mathrm{~s}^{-1}$.

Using the integrated line intensities in Table 2 (corrected by a beam-filling factor corresponding to a source size of $24^{\prime \prime}$, see Bergman et al. 2011), we performed a rotation diagram analysis (Goldsmith \& Langer 1999). From this we can determine the rotation temperature, $T_{\text {rot }}$, as well as the $\mathrm{HOOH}$ column density, $N(\mathrm{HOOH})$. The resulting rotation diagram is displayed in Fig. 4. The fit is based on the detected $\tau=2 \leftrightarrow 4$ lines. We derive $T_{\text {rot }}=22 \pm 3 \mathrm{~K}$ and a total $\mathrm{HOOH}$ column density of $(8 \pm 3) \times 10^{12} \mathrm{~cm}^{-2}$ where the errors depend on the uncertainty of the integrated intensities and a calibration uncertainty of $10-15 \%$. This rotation temperature is very close to the estimates of the kinetic temperatures from $\mathrm{H}_{2} \mathrm{CO}$ at the $\mathrm{SM} 1$ position. Obviously, the non-detection of the $\tau=1 \leftrightarrow 3$ lines (open squares in Fig. 4) is not consistent with the fit. Especially the $670 \mathrm{GHz}$ line should have been detected given these values of $T_{\text {rot }}$ and $N(\mathrm{HOOH})$. Hence, we conclude that the $\tau=1,3$ states are not populated according to a simple LTE model. The nondetection of the $670 \mathrm{GHz}$ line could be a result of subthermal excitation. The spontaneous rate coefficient is large (Table 1) and for a typical value of a collision coefficient $\left(\sim 10^{-10} \mathrm{~cm}^{3} \mathrm{~s}^{-1}\right)$, the critical density of the $670 \mathrm{GHz}$ transition is about two orders of magnitude higher than the $\mathrm{H}_{2}$ density determined from $\mathrm{H}_{2} \mathrm{CO}$ and $\mathrm{CH}_{3} \mathrm{OH}$ observations in the same source (Bergman et al. 2011). From the energy diagrams (Fig. 1) it is clear that there is a lack of radiative de-excitation routes out of the $K_{a}=0$ levels in the $\tau=1,3$ states (as opposed to the $\tau=2,4$ states). The population of the $\tau=1,3$ states could therefore be confined to the $K_{a}=0$ levels if the collisional excitation is inefficient. However, a full statistical equilibrium analysis is needed for understanding the details of the excitation. This also requires some basic knowledge of the collision coefficients. For now we assume that the $\tau=1,3$ population is negligible compared to the $\tau=2,4$ 


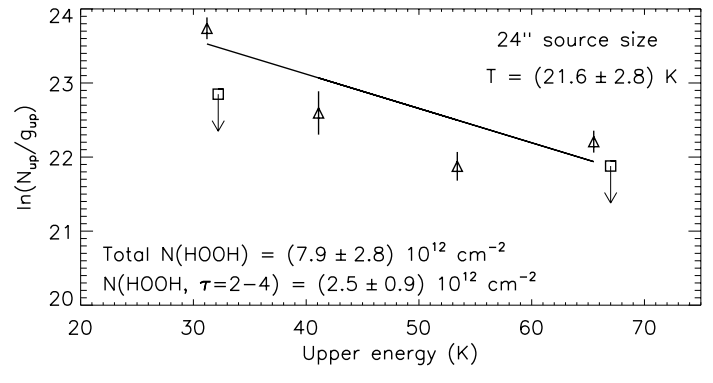

Fig. 4. HOOH rotation diagram. The detected lines (only $\tau=2 \leftrightarrow 4$ lines) are shown as open triangles with error bars. The size of the error bars corresponds to the total uncertainty (noise and calibration). The $3 \sigma$ upper limits (not included in the fit) are shown as open squares and downward arrows and originate from transitions between $\tau=1,3$ states. The fitted rotation temperature is noted, as is the total column density. The column density for unpopulated $\tau=1,3$ states is also given.

population. In this case, we instead find a total molecular column density of $(3 \pm 1) \times 10^{12} \mathrm{~cm}^{-2}$.

\section{Discussion}

Given the good agreement of the velocities of our detected $\mathrm{HOOH}$ lines (together with the $\approx 0.1 \mathrm{~km} \mathrm{~s}^{-1}$ accuracy of the laboratory frequencies) with those from other species we are very confident that the lines belong to $\mathrm{HOOH}$. From our mapping of the $219 \mathrm{GHz}$ line it is also evident that the NS velocity gradient seen for $\mathrm{HOOH}$ reflects that of other species. Moreover, the derived rotation temperature of $22 \pm 3 \mathrm{~K}$ is what would be expected for the $\rho$ Oph A cloud core. For this fairly low excitation temperature one would not expect many lines from other species to be present and, using the JPL and Cologne databases (Pickett et al. 1998; Müller et al. 2005), we found no lines from other species that could possibly interfere with the identification. Of course, the narrow lines (with $F W H M$ typically $<1 \mathrm{~km} \mathrm{~s}^{-1}$ ) seen toward the $\rho$ Oph A cloud core also make line confusion much less likely.

From the $\mathrm{H}_{2} \mathrm{CO}$ and $\mathrm{CH}_{3} \mathrm{OH}$ analysis of the SM1 core Bergman et al. (2011) determined an $\mathrm{H}_{2}$ column density of $3 \times 10^{22} \mathrm{~cm}^{-2}$. Assuming that the $\mathrm{HOOH}$ level populations mainly reside in the $\tau=2,4$ states, we arrive at an $\mathrm{HOOH}$ abundance of about $1 \times 10^{-10}$. This is well below the limit of $4.5 \times 10^{-10}$ found toward Orion KL by Blake et al. (1987).

According to current gas-phase schemes (e.g., the OSU chemical reaction database), formation of $\mathrm{HOOH}$ in the gas phase is not efficient. Only two very slow reactions are proposed for its formation, reaction of $\mathrm{H}_{2}$ with $\mathrm{HO}_{2}$, or reaction of two $\mathrm{OH}$ radicals.

On grains, $\mathrm{HOOH}$ is formed through successive hydrogen additions to $\mathrm{O}_{2}$. This was first proposed by Tielens \& Hagen (1982), based on theoretical arguments. Recent laboratory experiments have been made to investigate this route, up to formation of water molecules:

$\mathrm{O}_{2}+\mathrm{H}+\mathrm{H} \rightarrow \mathrm{HOOH}$

$\mathrm{HOOH}+\mathrm{H} \rightarrow \mathrm{H}_{2} \mathrm{O}+\mathrm{OH}$.

Miyauchi et al. (2008) have investigated the reaction of $\mathrm{H}$ atoms with solid $\mathrm{O}_{2}$ at $10 \mathrm{~K}$. Subsequently, Ioppolo et al. (2008) have investigated the same reaction in the temperature range $12-28 \mathrm{~K}$. Both studies showed that the conversion of $\mathrm{O}_{2}$ stops at some point before exhaustion of $\mathrm{O}_{2}$ because of shielding of $\mathrm{O}_{2}$ in the deepest layers. The experiment of Ioppolo et al. (2008) shows that the shielding decreases with increasing temperature, pointing to the fact that the $\mathrm{O}_{2}$ ice may become more porous when close to its sublimation temperature $(30 \mathrm{~K})$.

Because this shielding may not be very relevant in space, Oba et al. (2009) investigated the formation of $\mathrm{HOOH}$ and $\mathrm{H}_{2} \mathrm{O}$ when codeposing $\mathrm{O}_{2}$ and $\mathrm{H}$ in the temperature range $10-40 \mathrm{~K}$. The $\mathrm{H}_{2} \mathrm{O} / \mathrm{HOOH}$ ratio in the formed ices is observed to depend strongly on the temperature, and on the $\mathrm{O}_{2} / \mathrm{H}$ flux. The measured $\mathrm{H}_{2} \mathrm{O} / \mathrm{HOOH}$ ratio is lower than 5 in all experiments $(T=10$, $20 \mathrm{~K}$ and $\mathrm{O}_{2} / \mathrm{H}$-flux between $3.8 \times 10^{-4}$ and $1.9 \times 10^{-2}$ ), although higher values may be obtained in the case of lower $\mathrm{O}_{2} / \mathrm{H}$ flux. The present detection of $\mathrm{HOOH}$ may open the possibility to quantify the importance of reactions (1) and (2) in the formation of water.

Ioppolo et al. (2008) have modeled the formation of water in typical dense clouds (their Fig. 4). The laboratory results lead to a revision of the energy barriers involved in the models, and their new model (only accounting for the three main routes of water formation on the grains) predicts a fractional abundance for $\mathrm{HOOH}$ of a few $10^{-14}$ with respect to $\mathrm{H}$ nuclei. This is more than three orders of magnitude lower than our detection.

Further understanding will require detailed chemical modeling of grain chemistry. This will be the scope of a forthcoming paper. The observation of water in the $\rho$ Oph A region with the Herschel Observatory as well as the confirmation of the $\mathrm{O}_{2}$ detection would also be very valuable in setting constraints on the models.

Acknowledgements. We acknowledge the excellent observational support from the APEX staff. We are grateful to A. Gusdorf for doing some of the CHAMP ${ }^{+}$ observations. B.P. is funded by the Deutsche Forschungsgemeinschaft (DFG) under the Emmy Noether project number PA1692/1-1.

\section{References}

André, P., Ward-Thompson, D., \& Barsony, M. 1993, ApJ, 406, 122 Bergman, P., Parise, B., Liseau, R., \& Larsson, B. 2011, A\&A, 527, A39 Blake, G. A., Sutton, E. C., Masson, C. R., \& Phillips, T. G. 1987, ApJ, 315, 621 Boudin, N., Schutte, W. A., \& Greenberg, J. M. 1998, A\&A, 331, 749 Clancy, R. T., Sandor, B. J., \& Moriarty-Schieven, G. H. 2004, Icarus, 168, 116 Cohen, E. A., \& Pickett, H. M. 1981, J. Mol. Spectr., 87, 582

Encrenaz, T., Bézard, B., Greathouse, T. K., et al. 2004, Icarus, 170, 424

Goldsmith, P. F., \& Langer, W. D. 1999, ApJ, 517, 209

Güsten, R., Nyman, L. A., Schilke, P., et al. 2006, A\&A, 454, L13

Hartogh, P., Jarchow, C., Lellouch, E., et al. 2010, A\&A, 521, L49

Helminger, P., Bowman, W. C., \& de Lucia, F. C. 1981, J. Mol. Spect., 85, 120 Hougen, J. T. 1984, Can. J. Phys., 62, 1392

Hunt, R. H., Leacock, R. A., Wilbur Peters, C., \& Hecht, K. T. 1965, J. Chem. Phys., 42, 1931

Ioppolo, S., Cuppen, H. M., Romanzin, C., van Dishoeck, E. F., \& Linnartz, H. 2008, ApJ, 686, 1474

Kasemann, C., Güsten, R., Heyminck, S., et al. 2006, in SPIE Conf. Ser., 6275 Larsson, B., Liseau, R., Pagani, L., et al. 2007, A\&A, 466, 999

Liseau, R., Larsson, B., Brandeker, A., et al. 2003, A\&A, 402, L73

Liseau, R., Larsson, B., Bergman, P., et al. 2010, A\&A, 510, A98

Loren, R. B., Wootten, A., \& Wilking, B. A. 1990, ApJ, 365, 269

Miyauchi, N., Hidaka, H., Chigai, T., et al. 2008, Chem. Phys. Lett., 456, 27

Motte, F., André, P., \& Neri, R. 1998, A\&A, 336, 150

Müller, H. S. P., Schlöder, F., Stutzki, J., \& Winnewisser, G. 2005, J. Mol. Struc., 742,215

Oba, Y., Miyauchi, N., Hidaka, H., et al. 2009, ApJ, 701, 464

Pelz, G., Yamada, K. M. T., \& Winnewisser, G. 1993, J. Mol. Spect., 159, 507

Perrin, A., Flaud, J.-M., Camy-Peyret, C., et al. 1996, J. Mol. Spect., 176, 287

Petkie, D. T., Goyette, T. M. J. H. J., De Lucia, F. C., \& Helminger, P. 1995, J. Mol. Spect., 171, 145

Pickett, H. M., Poynter, R. L., Cohen, E. A., et al. 1998, J. Quant. Spec. Radiat. Transf., 60, 883

Tielens, A. G. G. M., \& Hagen, W. 1982, A\&A, 114, 245

Vassilev, V., Meledin, D., Lapkin, I., et al. 2008, A\&A, 490, 1157 